\title{
Statin Intolerance: Physiology and Treatment Alternatives
}

\author{
Sidney Carvalho Fernandes, Anita Saldanha, André Luis Valera Gasparoto, Ana Paula Pantoja, Henrique \\ Andrade Rodrigues da Fonseca and Tania Leme da Rocha Martinez*
}

Nephrology Department, BP - A Beneficência Portuguesa de São Paulo, Brazil

*Corresponding author: Tania Leme da Rocha Martinez, BP - A Beneficência Portuguesa de São Paulo, São Paulo, Brazil.

Received Date: November 28, 2019

Published Date: December 04, 2019

\begin{abstract}
Despite its proven effect on primary and secondary preventions of atherosclerotic disease, statins, which revolutionized the treatment of cardiovascular diseases, are still underutilized. One of the causes of this underuse lies in the fact that some patients have some kind of intolerance to this class of medications, and this intolerance may be related to clinical symptoms of myalgia and sometimes, in a small percentage, reaching severe cases of rhabdomyolysis. The therapeutic alternatives are discussed, highlighting the use of Oriza sativa fermented by Monascus purpureus. The use of Oryza extract fermented by Monascus purpureus is an appropriate alternative for the treatment of hypercholesterolemia in patients intolerant to statins.
\end{abstract}

Keywords: Intolerance to statins; Cholesterol treatment; Monascus purpureus; Myalgia; Myositis; Myopathy; Rhabdomyolysis

\section{Introduction}

Despite its proven effect on primary and secondary preventions of atherosclerotic disease, statins, which revolutionized the treatment of cardiovascular diseases, are still underutilized in this context, with a large percentage of patients who would benefit from their use and do not receive these drugs, with this estimated to occur in approximately $40 \%$ of patients eligible for this intervention [1]. One of the causes of this underuse lies in the fact that some patients have some kind of intolerance to this class of medications, and this intolerance may be related to clinical symptoms of myalgia and sometimes, in a small percentage, coming to severe cases of rhabdomyolysis, digestive symptoms such as epigastric pain, heartburn, constipation, flatulence, nausea or vomiting, dyspepsia, headache, rinitis, sinusitis and sometimes raising the level of liver enzymes. Because hypercholesterolemia is an asymptomatic condition, the unpleasant side effects of the drugs used for its treatment end up greatly decreasing adherence to this. In addition, patients generally do not have a very real view of how hypercholesterolemia increases cardiovascular risk, especially in cases of primary prevention. It is noteworthy that these symptoms and signs are much more frequent when there is an association with other drugs used in the treatment of dyslipidemias, such as niacin and fibrates. It is noteworthy here that, of fibrates, genfibrozil is the one that presents the most drug interaction with statins, this occurring by two of its pharmacokinetic characteristics: first ly because it competes with the same glucoronil transferase that metabolizes most statins; secondly because it inhibits an important hepatocyte membrane conveyor, oatp2 (organic anion transporter protein) which is the carrier responsible for internalizing statins in the oxytor (with difficulty in statins entering in hepatocytes, their serum level increases) [2]. Also these undesirable effects are more frequent in real clinical practice than the incidence presented in large clinical studies, where there is a pre-selection of patients.

\section{Statin vs. Myalgia}

Of all the side effects mentioned above the most common and greatest responsible for suspending the use of statins is myalgia. The occurrence of this is related to the dose of the drug used and characteristically occurs in the absence of myositis. It is also very common to occur myalgia at the beginning of treatment, when patients are instructed to start a physical exercise program and concomitantly prescribes a statin. For this reason it is our conduct to start first recommending the practice of exercises and only a few days after the beginning of this is that we prescribe statin. Before 
entering the pathophysiology of myalgias it is useful to establish diagnostic criteria of the various conditions known as statinrelated myopathy. We adopted the criteria of the American Heart Association/American College of Cardiology/National Heart, Lung and Blood Institute [3].

Myopathy: general term referring to any muscle disease.

Myalgia: muscle pain or weakness without elevation of creatinokinase.

Myositis: muscle symptoms with elevation of creatinokinase.

Rhabdomyolysis: muscle symptoms with increased creatinokinase above 10 times the upper normal level, elevation of creatinine, usually with dark urine with urinary myoglobin.

\section{Pathophysiology of Myalgia}

The basic mechanism of myopathy is also discussed, and the following are proposed: instability of the membrane of myocytes by decreasing its cholesterol content, depletion of isoprenoids or Coenzyme Q10 and mitochondrial dysfunction. The first mechanism does not seem to be correct, because decreased cholesterol synthesis by scalen sintetase inhibitors does not result in myopathy. As for the depletion of coenzyme Q10 and mitochondrial dysfunction, the various studies are still contradictory [4]. Some recent pharmacogenetic studies suggest that myopathy associated with statin use is genetically determined and is related to polymorphisms in genes responsible for coenzyme Q10 synthesis, especially the COQ2 gene [5-8].

\section{Risk Factors for Myalgia}

The risk factors for the onset of myopathy are: patients with complex clinical situations, using polypharmacy, hypothyroidism, women with low weight, intense physical activity, hospitalization for large surgeries, patients geriatric drugs, use of high doses of statins and drug interaction with CYP 3A4 inhibitordrugs, mainly with azole alour antigens (ketoconazole and itraconazole - alternatively use fluconazole), macroliteic antibiotics (erythromycin and clarithromycin), protease inhibitors, cyclosporine, amiodarone and, as already described above, with genfibrozil.

\section{Therapeutic Suggestion for Statin Intolerant}

Several treatment strategies have been suggested for use in case of statin intolerance:

Coenzyme Supplementation Q10: as it has been suggested that one of the causes of myopathy would be coenzyme Q10 deficiency in the myocyte membrane, some studies evaluated their supplementation in these cases. The results were contradictory and a systematic review was inconclusive [9]. Coenzyme Q10 per day concomitantly with statin has been used 100 to $200 \mathrm{mg}$ per day, with a reasonable improvement in tolerance.

Statin change: use of other statins, such as rosuvastatin that is minimally metabolized, pravastatin that is not metabolized by cytochrome P450 and fluvastatin that is metabolized by the passage of 2C9. However this change would only be indicated in cases of drug associations. Also with the use of fluvastatin alone, about $17 \%$ of patients had recurrence of myopathy [10]. It is also recommended the use of atorvastatin or rosuvastatin in low doses and in non-daily treatment schemes: the use of these 2 potent statins, of longer effect, in low doses and in 2 to 3 weekly doses, proved to be efficient in reducing the LDL-cholesterol from 13 to $25 \%$, with good tolerance; but studies are lacking that show the improvement of clinical outcomes with these regimens.

Use of ezethyiba: although ezethycitis can, when used in isolation, reduce LDL-cholesterol levels by up to $20 \%$, its efficacy in reducing clinical outcomes has not yet been demonstrated. Ezethyiba is useful when used together with statins.

Use of bile acid sequeblant resins: the use of these resins is efficient in reducing LDL-cholesterol by about $20 \%$, but there is a difficulty in adherence to treatment due to its unpleasant side effects, such as impalatability, intestinal constipation and also interference in the absorption of other drugs. In addition, the use of these medicines may raise the serum level of VLDL.

Use of fermented red yeast rice extract (red yeast rice-extract of Oryza sativa fermented by Monascus purpureus): such extract is a herbal plant that has been used in China since the 800s AD, for various purposes, among them the treatment of diseases of the circulatory system. Currently this product is already produced in a standardized way, registered as a medicine by the National Health Surveillance Agency and contains, in capsules of $600 \mathrm{mg}$, monacolin $\mathrm{K}$ (lovastatin) and 8 other monacolins, in addition to unsaturated fats and other Substances. This product was clinically tested in a large clinical secondary prevention study involving 4,870 patients who had already suffered a myocardial infarction, with an average of 60.5 years (18 to 70 years), with a follow-up of 4.5 years and a dose of $600 \mathrm{mg}$ of the extract, tested against placebo. This study showed a favorable outcome, with statistical significance, in the following parameters: total mortality, cardiovascular mortality, coronary mortality, cancer mortality, non-fatal myocardial infarction and revascularization coronary artery, and such events are also favorable in the subgroups of diabetics and the elderly [11].

Due to these clinical findings, this product has been tested against placebo in a study involving statin-intolerant patients. The patients were randomized and allocated into two groups (placebo and treatment) and both received dietary guidance and for physical exercise. Additionally, patients in the treated group received 1800 $\mathrm{mg} /$ day of the extract. The treatment group showed a statistically significant reduction in LDL-C (-21.3\% vs. $-8.7 \%)$, total cholesterol $(-14.9 \%$ vs. $-5.3 \%)$ triglycerides $(-7.2 \%$ vs. $-1.4 \%)$ compared to the placebo group. The appearance of myalgias and alteration of CPK and liver enzymes was not different between groups [12-15]. A recent systematic review showed that this extract presented a good regulatory effect of lipidemia and was also safe and effective in reducing cardiovascular events in patients with coronary disease complicated with dyslipidemia16. In addition to this effect on lipids, this extract showed a favorable effect on several parameters related to metabolic syndrome and obesity, such as decreased 
insulin resistance, serum level of this and also adiponectin and improvement of non-alcoholic steatohepatitis $[17,18]$. Therefore, the use of Oryza extract fermented by Monascus purpureus is an excellent alternative for the treatment of hypercholesterolemia in patients intolerant to statins.

\section{Conclusion}

Despite all this discussion on pharmacological agents, let us not forget that in the treatment of dyslipidemias is fundamental and should always come first the implementation of hygiene measures, with the performance of regular physical exercises and a diet with low saturated fats, sugar and sweets, aiming at improving lipid levels and correcting excess weight when present. It is also worth remembering that phytosterolis, both in pharmaceutical formulation or in the form of functional foods, if ingested at a minimum dose of two daily outlets, can reduce LDL levels from 7 to $10 \%$.

\section{Acknowledgments}

None.

\section{Conflicts of Interest}

No conflict of interest.

\section{References}

1. Mitka M (2003) Expanding statin use to help more at-risk patients is causing financial heartburn. JAMA 290(17): 2243-2245.

2. Mahley RW, Bersot TP (2006) Drug therapy for hypercholesterolemia and dyslipidemia. In: Brunton LL, Lazo JS, Parker KL (Eds.), Goodman \& Gilman's The Pharmacological Basis of Therapeutics. $11^{\text {th }}($ Edn.), McGraw-Hill New York, pp. 933-966.

3. Pasternak RC, Smith SC Jr, Bairey-Merz CN, Grundy SM, Cleeman JI, et al. (2002) ACC/AHA/NHLBI clinical advisory on the use and safety of statins. J Am Coll Cardiol 40(3): 567-572.

4. Joy TR, Hegele RA (2009) Narrative review: statin-related myopathy. Ann Intern Med 150(12): 858-868.

5. Oh J, Ban MR, Miskie BA, Pollex RL, Hegele RA, et al. (2007) Genetic determinants of statin intolerance. Lipids Health Dis 6: 7.
6. Stroes ES, Thompson PD, Corsini A, Vladutiu GD, Raal FJ, et al. (2015) Statin-associated muscle symptoms: impact on statin therapy-European Atherosclerosis Society Consensus Panel Statement on Assessment, Aetiology and Management. Eur Heart J 36(17): 1012-2022.

7. Alonso R, Cuevas A, Cafferata A (2019) Diagnosis and management of statin intolerance. J Atheroscler Thromb 26(3): 207-215.

8. Toth PP, Patti AM, Giglio RV, Nikolic D, Castellino G, et al. (2018) Management of statin intolerance in 2018: still more questions than answers. Am J Cardiovasc Drugs 18(3): 157-173.

9. Marcoff L, Thompson PD (2007) The role of coenzyme Q10 in statinassociated myopathy: a systematic review. J Am Coll Cardiol 49(23): 2231-2237.

10. Stein EA, Ballantyne CM, Windler E, Sirnes PA, Sussekov A, et al. (2008) Efficacy and tolerability of fluvastatin XL $80 \mathrm{mg}$ alone, ezetimibe alone, and the combination of fluvastatin XL $80 \mathrm{mg}$ with ezetimibe in patients with a history of muscle-related side effects with other statins. Am J Cardiol 101(4): 490-496.

11. Lu Z, Kou W, Du B, Wu Y, Zhao S, et al. (2008) Effect of Xuezhikang, an extract from red yeast Chinese rice, on coronary events in a Chinese population with previous myocardial infarction. Am J Cardiol 101(12): 1689-1693.

12. Becker DJ, Gordon RY, Halbert SC, French B, Morris PB, et al. (2009) Red yeast rice for dyslipidemia in statin-intolerant patients: a randomized trial. Ann Intern Med 150(12): 830-839.

13. Santini A, Novellino E (2017) Nutraceuticals in hypercholesterolaemia: an overview. Br J Pharmacol 174(11): 1450-1463.

14. Ogawara $H$ (2018) Comparison of strategies to overcome drug resistance: learning from various kingdoms. Molecules 23(6): E1476.

15. Kim D, Ku S (2018) Beneficial effects of monascus sp. KCCM 10093 pigments and derivatives: a mini review. Molecules 23(1): E98.

16. Shang Q, Liu Z, Chen K, Xu H, Liu J, et al. (2012) A systematic review of xuezhikang, an extract from red yeast rice, for coronary heart disease complicated by dyslipidemia. Evid Based Complement Alternat Med 2012: 636547.

17. Fujimoto M, Tsuneyama K, Chen SY, Nishida T, Chen JL, et al. (2012) Study of the effects of monacolin $\mathrm{k}$ and other constituents of red yeast rice on obesity, insulin-resistance, hyperlipidemia, and nonalcoholic steatohepatitis using a mouse model of metabolic syndrome. Evid Based Complement Alternat Med 2012: 892697.

18. Affuso F, Mercurio V, Ruvolo A, Pirozzi C, Micillo F, et al. (2012) A nutraceutical combination improves insulin sensitivity in patients with metabolic syndrome. World J Cardiol 4(3): 77-83. 